\title{
Penetration depth study of very thin superconducting Nb films
}

\author{
Thomas R. Lemberger,* Iulian Hetel, Jacob W. Knepper, and F. Y. Yang \\ Department of Physics, The Ohio State University, 191 W. Woodruff Ave., Columbus, Ohio 43210-1117, USA
}

(Received 22 March 2007; revised manuscript received 29 May 2007; published 20 September 2007)

\begin{abstract}
Using a low-frequency two-coil technique, we measure the magnetic penetration depth $\lambda(T)$ of superconducting Nb films with thicknesses $20 \AA \leqslant d \leqslant 228 \AA$ sputtered onto oxidized Si substrates. We find a phenomenological dependence of $T_{c}$ on $d, T_{c} / 8.5 \mathrm{~K} \approx \tanh (d / 70 \AA)$ for films thinner than $250 \AA . \lambda^{-2}(T) / \lambda^{-2}(0)$ is well fitted by weak-coupling dirty-limit theory with a weak-coupling gap, $\Delta(0)=1.8 k_{B} T_{c} . \lambda^{-2}(0)$ agrees with dirty-limit theory, given the experimental values of transition temperature $T_{c}$ and residual resistivity $\rho_{0}$. These results indicate that the suppression of $T_{c}$ is due to mechanisms that weaken the effective pairing interaction and not due to pair breaking interactions.
\end{abstract}

DOI: 10.1103/PhysRevB.76.094515

PACS number(s): 74.25.Nf, 74.78.Db, 74.62.-c, 74.25.Fy

\section{INTRODUCTION}

\section{A. Background}

Gubin et al. ${ }^{1}$ recently reported the first systematic study of the magnetic penetration depth $\lambda$ of superconducting $\mathrm{Nb}$ films. They measured films as thin as $80 \AA$ dc sputtered onto $\mathrm{Si}$ substrates. In agreement with numerous previous studies, Gubin et al. found that resistivity goes up and superconducting transition temperature goes down as $\mathrm{Nb}$ film thickness $d$ decreases. They were able to establish that $\lambda(0)$ increases as $d$ decreases in quantitative agreement with dirty-limit BCS theory, ${ }^{2}$ given the measured $T_{c}$ and resistivity. They detected small amounts of carbon on their $\mathrm{Nb}$ film surfaces, and hypothesized that surface contaminants result in thin normal layers on the top and bottom of sputtered $\mathrm{Nb}$ films, weakening the average pairing interaction and thereby contributing to the reduction of $T_{c}$ while preserving the applicability of BCS theory. Another possibility is that dc sputtering produces films with surface or volume impurities that are magnetic, and the observed reduction in $T_{c}$ is due in part to magnetic pair breaking. ${ }^{3}$ Differences between these two possibilities grow as $d$ and $T_{c}$ decrease, motivating the present study of thinner $\mathrm{Nb}$ films, down to $20 \AA$.

The present work extends Gubin et al. ${ }^{1}$ by studying thinner films and by providing a more detailed study of the $T$ dependence of $\lambda$ designed to see whether deviations from dirty-limit BCS theory emerge in films thinner than $80 \AA$. On a technical level, our work complements Gubin et al. in that we measure $\lambda$ with a different, low-frequency method. The fact that we obtain essentially the same values for $\lambda(0)$ for films of similar thickness bolsters our confidence in the accuracy of our measurements and the lab-to-lab and day-today reproducibility of dc sputtered $\mathrm{Nb}$ films. There have been a number of studies of thin $\mathrm{Nb}$ films. Of particular note is the tunneling study of Park and Geballe ${ }^{4}$ of $\mathrm{Nb}$ films as thin as $9 \AA$, which were electron-beam evaporated onto sapphire substrates using a protocol that eliminated carbon contamination. They found a clean BCS-like gap in the electron density of states, indicating the absence of pair breaking. Our sputtered films will be compared with e-beam films below.

\section{B. Theory}

In dirty-limit theory for superconductivity, where the elastic scattering rate $1 / \tau$ is much larger than the superconduct- ing gap $\Delta(0) / \hbar$, the magnitude and $T$ dependence of $\lambda$ are simple to express. $\lambda^{-2}(T)$ has the form ${ }^{2}$

$$
\frac{\lambda^{-2}(T)}{\lambda^{-2}(0)}=\frac{\Delta(T)}{\Delta(0)} \tanh \left[\frac{\Delta(T)}{2 k_{B} T}\right],
$$

where $k_{B}$ is Boltzmann's constant, and the normalized gap $\Delta(T) / \Delta(0)$ is approximated by ${ }^{5}$

$$
\frac{\Delta(T)}{\Delta(0)} \approx\left[\cos \left(\frac{\pi T^{2}}{2 T_{c}^{2}}\right)\right]^{1 / 2} .
$$

$\lambda^{-2}(0)$ is reduced by scattering such that its magnitude is inversely proportional to residual resistivity $\rho_{0}$,

$$
\left.\lambda^{-2}(0)\right|_{B C S}=\frac{\pi \mu_{0} \Delta(0)}{\hbar \rho_{0}} .
$$

Here, $\mu_{0}$ is the permeability of vacuum. We measure $\lambda^{-2}(0)$ and $\rho_{0}$ and obtain $\Delta(0)$ from fitting $\lambda^{-2}(T)$, so we can test whether dirty-limit BCS applies to our films.

Now we consider what to expect if most of the suppression of $T_{c}$ is due to a pair breaking interaction. ${ }^{3}$ These interactions cause significant deviations from BCS theory. In particular, the peak in the density of states at $E \approx \Delta$ is broadened, and the gap edge shifts down in energy. The density of states is gapless when the pair breaking energy $\hbar / \tau_{\mathrm{pb}}$ exceeds $\Delta$. The pair breaking energy $\hbar / \tau_{\mathrm{pb}}$ can be estimated from the suppression of $T_{c}$ below the $9.288 \mathrm{~K}$ transition temperature $^{6}$ of bulk $\mathrm{Nb}$. For present purposes, we can use the approximate result, ${ }^{3}$

$$
k_{B}\left(9.3 \mathrm{~K}-T_{c}\right) \approx \frac{\pi \hbar}{4 \tau_{\mathrm{pb}}} .
$$

As pair breaking lowers the gap edge in the density of states, $\lambda^{-2}(0)$ decreases below its BCS value [Eq. (3)], ${ }^{3}$

$$
\frac{\lambda^{-2}(0)}{\left.\lambda^{-2}(0)\right|_{B C S}} \approx 1-0.42 \frac{\hbar}{\tau_{\mathrm{pb}} \Delta(0)} \quad\left[\hbar / \tau_{\mathrm{pb}} \Delta(0) \leqslant 1\right] .
$$

For example, a $d=32 \AA$ film has $T_{c} \approx 3.9 \mathrm{~K}$. Equation (4) then predicts $\hbar / k_{B} \tau_{\mathrm{pb}} \approx 3.5 \mathrm{~K}$, assuming that half of the suppression of $T_{c}$ is due to pair breaking. The order parameter can be estimated as $\Delta(0) / k_{B} \approx 2 T_{c} \approx 7.8 \mathrm{~K}$. Equation (5) tells us to expect a $20 \%$ deviation below BCS in this case. The $T$ 
dependence of $\lambda^{-2}$ should be affected as well. Pair breaking softens the exponentially flat low- $T$ behavior in BCS theory until, in the gapless limit, $\hbar / \tau_{\mathrm{pb}}>\Delta(0) ; \lambda^{-2}(T)$ is approximately quadratic,

$$
\frac{\lambda^{-2}(T)}{\lambda^{-2}(0)} \approx 1-\frac{T^{2}}{T_{c}^{2}} \quad\left[\hbar / \tau_{\mathrm{pb}} \Delta(0)>1\right] .
$$

The foregoing shows that if a significant portion of the decrease in $T_{c}$ is due to pair breaking, the thinnest films may exhibit deviations from BCS theory, large enough to be discerned experimentally.

\section{EXPERIMENTAL DETAILS}

Films are dc sputtered from a 2 in. diameter $\mathrm{Nb}$ target onto oxidized $\mathrm{Si}$ substrates 2 in. above the target. A protective layer of Ge $200 \AA$ thick is dc sputtered immediately after the $\mathrm{Nb}$ deposition. Deposition rates are 1.5 and $2.0 \AA / \mathrm{s}$, respectively. Substrates nominally $18 \times 18 \times 0.4 \mathrm{~mm}^{3}$ are placed in a load-locked UHV chamber with a background pressure of $5 \times 10^{-10}$ torr. Substrates are nominally at room temperature during deposition. Film thickness is calibrated by growing a thick film, measuring its thickness with an atomic force microscope, and thereafter assuming that film thicknesses are proportional to sputtering time. We checked that films with the same nominal thickness grown in separate runs have very similar properties.

The sheet conductivity $Y \equiv \sigma_{1} d-i \sigma_{2} d$ is measured at $50 \mathrm{kHz}$ using a two-coil mutual inductance technique in which coaxial drive and pickup coils are pressed against opposite sides of the center of the sample film. ${ }^{7,8}$ The usual conductivity $\sigma_{1}-i \sigma_{2}$ can be obtained from $Y / d$ if the film thickness $d$ is known. The coils are solenoids nominally $2 \mathrm{~mm}$ in diameter and $2 \mathrm{~mm}$ in length, designed to be much smaller than the areal dimensions of the films. Induced currents lie in the plane of the sample. The ac magnetic field generated by the $50 \mathrm{kHz}$ current in the drive coil is kept low enough that $Y$ is independent of the ac field amplitude. Reducing the applied ac field by a factor of 10 has no effect on the data. The ambient dc magnetic field is reduced by a double $\mu$-metal shield around the He-4 cryostat.

From the mutual inductance measured with the sample present, we subtract the mutual inductance measured with a thick $(0.1 \mathrm{~mm})$ superconducting $\mathrm{Pb}$ foil in place of the sample. This adjustment removes coupling due to magnetic flux that goes around the sample film as well as stray coupling from other parts of the circuitry. It permits us to proceed as if the films were infinite in area. ${ }^{7,8} \mathrm{We}$ analyze the normalized mutual inductance, i.e., the ratio of the adjusted mutual inductance to the mutual inductance above $T_{c}$, where the sample is undetectable. Normalization cancels out small run-to-run variations in substrate thickness, alignment of coils, etc. To illustrate how all this plays out, consider the worst case. Our thickest film, $d=228 \AA$, screens the mutual inductance from about $210 \mathrm{nH}$ above $T_{c}$ to about $300 \mathrm{pH}$ at $T \ll T_{c}$. We measured that a $\mathrm{Pb}$ foil with the same areal dimensions as the sample film screens from $210 \mathrm{nH}$ to $44 \pm 10 \mathrm{pH}$. Thus, we deduce that coupling through the sample film is $256 \pm 10 \mathrm{pH}$ at low $T$. The $\pm 10 \mathrm{pH}$ uncertainty in the $\mathrm{Pb}$ foil measurement contributes an uncertainty of $\pm 4 \%$ in $Y$. At higher temperatures and for thinner films, coupling through the film is larger than $256 \mathrm{pH}$, so the $\pm 10 \mathrm{pH}$ uncertainty contributes a proportionally smaller uncertainty to $Y$.

\section{EXPERIMENTAL RESULTS}

We measure resistivity by cutting the substrate into an approximately $2 \times 12 \mathrm{~mm}^{2}$ rectangle, then attaching wires via pressed indium pads. Within the crude experimental uncertainty of about $20 \%$, the residual resistivities of our films agree with the phenomenological expression of Gubin et al., ${ }^{1}$

$$
\rho_{0} \approx\left(3.7+\frac{1500 \AA}{d}\right) \mu \Omega \mathrm{cm},
$$

down to $d=32 \AA$. The dependence of $\rho_{0}$ on $d$ is due to surface scattering. Residual resistivity rises faster than this expression predicts when $d<32 \AA$. A similar deviation is observed in e-beam deposited films. ${ }^{4}$ To minimize "noise," we use the phenomenological expression for $d \geqslant 32 \AA$ and our measured value for $d<32 \AA$.

Our films are in the dirty limit, $\ell \ll \xi_{0}$, where $\ell=v_{F} \tau$ is the electron mean free path for elastic scattering, and $\xi_{0}$ $=\hbar v_{F} / \pi \Delta(0)$ is the superconducting coherence length, as we now show. $v_{F}$ is the Fermi velocity. The value ${ }^{9} \rho_{0} \ell=3.72$ $\times 10^{-6} \mu \Omega \mathrm{cm}^{2}$ is commonly used ${ }^{1,4}$ to estimate the electron mean free path of thin $\mathrm{Nb}$ films, although its use relies on certain assumptions regarding the similarity of band structure of thin films and bulk Nb. With Eq. (7), $\ell$ is less than $100 \AA$,

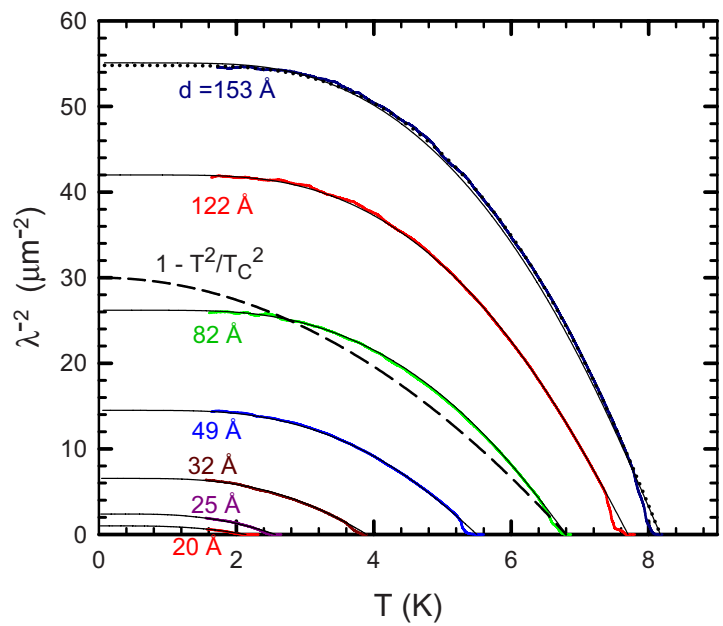

FIG. 1. (Color online) $\lambda^{-2}(T)$ vs $T$ for $\mathrm{Nb}$ films with various thicknesses. Dirty-limit BCS theory (thin solid black curves) fits the data well. Best fit values of $\alpha \equiv \Delta(0) / k_{B} T_{c}$ are 1.80 for thinner films and perhaps 1.90 for thicker films, $d \geqslant 153 \AA$. Dashed curve is the quadratic predicted by pair breaking theory for gapless superconductors. 


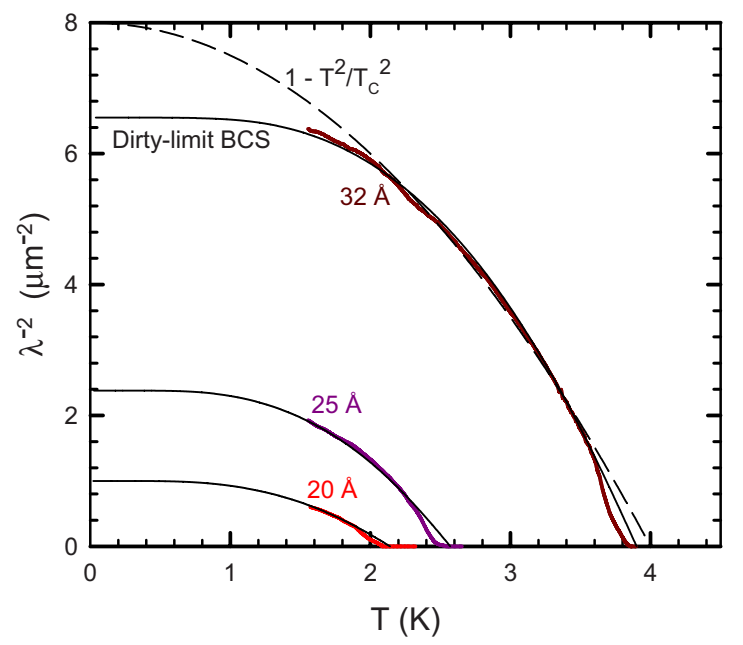

FIG. 2. (Color online) $\lambda^{-2}(T)$ vs $T$ for three thinnest $\mathrm{Nb}$ films. BCS theory (thin solid curves) fits data for $d=32 \AA$, whereas a quadratic (thin dashed curve) does not.

$$
\ell \approx \frac{100 \AA}{1+400 \AA / d} .
$$

With $v_{F} \approx 6 \times 10^{5} \mathrm{~m} / \mathrm{s},{ }^{9}$ and $\Delta(0) \approx 2 k_{B} T_{c}$, we estimate a clean-limit coherence length, $\xi_{0}=\hbar v_{F} / \pi \Delta(0)>1000 \AA$. Hence, we are confident that our films are well into the dirty limit. The disorder-shortened coherence length, $\xi \approx\left(\xi_{0} \ell\right)^{1 / 2}$, is larger than $d$, so our films are two-dimensional superconductors.

From $\sigma_{2} d$, we obtain the ratio of areal superfluid density $n_{S} d$ to effective mass of superconducting electrons: $n_{S} d / m^{*}$ $=\omega \sigma_{2} d / e^{2}$, where $e$ is the electronic charge. It is conventional to present the results in terms of the inverse magnetic penetration depth squared $\lambda^{-2}(T)$, which is proportional to $n_{S} / m^{*}, d / \lambda^{2} \equiv \mu_{0} \omega \sigma_{2} d$. We believe that our method determines $d / \lambda^{2}$ to $<3 \%$ accuracy for films thinner than $200 \AA$, and $1 / \lambda^{2}$ to about $\pm 5 \%$ accuracy, with the extra uncertainty

TABLE I. Nb film parameters. Residual resistivity $\rho_{0}$ is calculated from Eq. (7) for $d \geqslant 32 \AA$ and measured for $d=20$ and $25 \AA$. Residual sheet resistance, $R_{N} \equiv \rho_{0} / d$. We could not determine a meaningful value for $\Delta(0) / k_{B} T_{c}$ for the $20 \AA$ thick film. $\left.\lambda^{-2}(0)\right|_{B C S}$ is calculated from Eq. (3) with experimental values for $\Delta(0)$ and $\rho_{0}$.

\begin{tabular}{|c|c|c|c|c|c|c|}
\hline $\begin{array}{l}d \\
(\AA)\end{array}$ & $\begin{array}{c}T_{c} \\
(\mathrm{~K}) \\
( \pm 0.1 \mathrm{~K})\end{array}$ & $\begin{array}{c}2 \Delta(0) / k_{B} T_{c} \\
\quad( \pm 0.05)\end{array}$ & $\begin{array}{c}\rho_{0} \\
(\mu \Omega \mathrm{cm}) \\
( \pm 10 \%)\end{array}$ & $\begin{array}{l}R_{N} \\
(\Omega)\end{array}$ & $\begin{array}{c}\lambda^{-2}(0) \\
\left(\mu \mathrm{m}^{-2}\right) \\
( \pm 5 \%)\end{array}$ & $\begin{array}{c}\left.\lambda^{-2}(0)\right|_{B C S} \\
\left(\mu \mathrm{m}^{-2}\right)\end{array}$ \\
\hline 20 & 2.14 & $\cdots$ & 153 & 760 & $1.0 \pm 0.25$ & 1.2 \\
\hline 25 & 2.57 & 1.80 & 100 & 400 & 2.38 & 2.4 \\
\hline 32 & 3.90 & 1.80 & 51 & 160 & 6.55 & 7.1 \\
\hline 49 & 5.50 & 1.80 & 34 & 69 & 14.3 & 15 \\
\hline 82 & 6.80 & 1.80 & 22 & 27 & 26.2 & 29 \\
\hline 122 & 7.70 & 1.80 & 16 & 13 & 42.0 & 45 \\
\hline 153 & 8.17 & 1.90 & 13.5 & 9 & 54.8 & 56 \\
\hline 228 & 8.45 & 1.90 & 10.3 & 4.5 & 73.0 & 76 \\
\hline
\end{tabular}

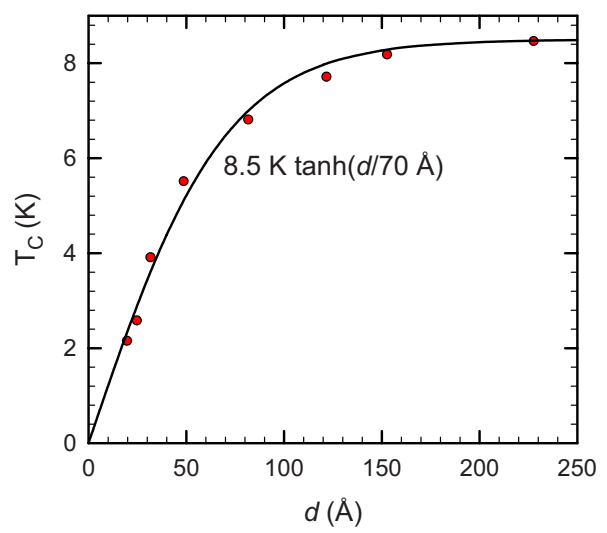

FIG. 3. (Color online) $T_{c}$ vs $d$ for sputtered $\mathrm{Nb}$ films. Black curve is an empirical fit valid for $d<250 \AA$.

arising from the roughly $4 \%$ uncertainty in $d$.

The main contribution of this paper is the set of $\lambda^{-2}(T)$ vs $T$ curves in Figs. 1 and 2, together with the film parameters in Table I. The thin solid curves in Figs. 1 and 2 are dirtylimit fits with a weak-coupling value of $\alpha \equiv \Delta(0) / k_{B} T_{c}$ $=1.80$. The fits are good, and they yield values for $\lambda^{-2}(0)$ and the mean-field transition temperature $T_{c}$. Figure 3 shows the dependence of $T_{c}$ on $d$. For the thickest films, it appears that a slightly larger value, $\alpha=1.90$ (dotted curve), fits a little better, suggesting that electron-phonon coupling is stronger in films thicker than $150 \AA$ or so. For comparison, quadratic "fits" (dashed) are shown for the 82 and $32 \AA$ films. The $32 \AA$ film is the thinnest film for which we have data over a wide enough temperature range to distinguish that the BCS fit is better than the quadratic. However, even though the $T$ dependence of $\lambda^{-2}(T)$ for the 20 and $25 \AA$ films can be fitted equally well by either theory, the value of $\lambda^{-2}(0)$ obtained from the BCS fit is consistent with the BCS theory [Eq. (3) and Table I], whereas the value of $\lambda^{-2}(0)$ obtained from the quadratic fit is larger than the BCS prediction, and it would have to be smaller to be consistent with pair breaking theory.

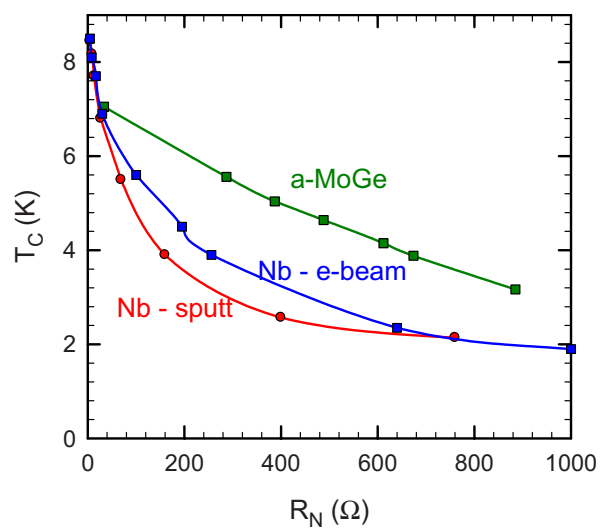

FIG. 4. (Color online) $T_{c}$ vs sheet resistance $R_{N}$ for sputtered $\mathrm{Nb}$ films, e-beam evaporated $\mathrm{Nb}$ films (Ref. 4), and amorphous MoGe films (Ref. 13). Curves are guides to the eye. The six $a$-MoGe films with $200 \Omega<R_{N}<1000 \Omega$ have thicknesses from 21.5 to $61 \AA$, comparable to the five thinnest sputtered $\mathrm{Nb}$ films. 
Hence, BCS dirty-limit theory provides a consistent description of our data.

As an aside, we note that $\lambda^{-2}(T)$ consistently shows an abrupt downturn as $T$ approaches $T_{c}$. We believe that this is due to inhomogeneity in the films, although we have worked very hard to optimize film homogeneity. The downturn is not the vortex-pair unbinding transition expected in twodimensional superconductors because it occurs at a superfluid density at least a factor of 10 higher than the theory predicts. Slight inhomogeneity of the films does not affect conclusions of this paper.

A comparison between $\mathrm{Nb}$ films grown by dc sputtering and e-beam evaporation is interesting. E-beam films consistently have higher $T_{c}$ 's for a given film thickness. A large part of the difference can attributed to the lower resistivities of e-beam films. When we plot $T_{c}$ vs residual sheet resistance, $R_{N}=\rho_{0} / d$ (Fig. 4), we find that the two types of films are not so different. This particular plot is motivated by the fact that in strongly disordered thin films, $T_{c}$ is expected to decrease roughly linearly with $R_{N}$ due to diminished screening of the Coulomb interaction between electrons. ${ }^{10}$ For context, Fig. 4 shows that amorphous MoGe films display the predicted behavior. $^{11-14}$

\section{CONCLUSION}

We examined the magnetic penetration depth of thin superconducting $\mathrm{Nb}$ films dc sputtered onto oxidized $\mathrm{Si}$ substrates at room temperature. $T_{c}$ is approximately linear in film thickness $d$ for $20 \AA \leqslant d \leqslant 70 \AA$. The magnitude and $T$ dependence of $\lambda^{-2}(T)$ indicate that these films are weakcoupling dirty-limit BCS superconductors, implying that $T_{c}$ decreases due to non-pair-breaking mechanisms, e.g., an increase in Coulomb pseudopotential. ${ }^{4}$ These results are consistent with tunneling measurements of electron-beam deposited $\mathrm{Nb}$ films, ${ }^{4}$ when films are compared on the basis of sheet resistance rather than thickness.

\section{ACKNOWLEDGMENT}

I.H. gratefully acknowledges support from OSU.

*Author to whom correspondence should be addressed.

${ }^{1}$ A. I. Gubin, K. S. Il'in, S. A. Vitusevich, M. Siegel, and N. Klein, Phys. Rev. B 72, 064503 (2005).

${ }^{2}$ M. Tinkham, Introduction to Superconductivity (McGraw-Hill, New York, 1975).

${ }^{3}$ See the review by K. Maki, in Superconductivity, edited by R. D. Parks (Dekker, New York, 1969), Vol. 2, Chap 18.

${ }^{4}$ S. I. Park and T. H. Geballe, Phys. Rev. Lett. 57, 901 (1986).

${ }^{5}$ T. P. Sheahan, Phys. Rev. 149, 368 (1966).

${ }^{6}$ B. Fellmuth, H. Maas, and D. Elefant, Metrologia 21, 169 (1985).

${ }^{7}$ S. J. Turneaure, E. R. Ulm, and T. R. Lemberger, J. Appl. Phys. 79, 4221 (1996).

${ }^{8}$ S. J. Turneaure, A. A. Pesetski, and T. R. Lemberger, J. Appl. Phys. 83, 4334 (1998).

${ }^{9}$ A. F. Mayadas, R. B. Laibowitz, and J. J. Cuomo, J. Appl. Phys. 43, 1287 (1972).

${ }^{10}$ A. Finkel'shtein, JETP Lett. 45, 46 (1987); Physica B 197, 636 (1994).

${ }^{11}$ J. M. Graybeal, Physica B \& C 135, 113 (1985).

${ }^{12}$ J. M. Graybeal and M. R. Beasley, Phys. Rev. B 29, 4167 (1984).

${ }^{13}$ S. J. Turneaure, T. R. Lemberger, and J. M. Graybeal, Phys. Rev. Lett. 84, 987 (2000).

${ }^{14}$ S. J. Turneaure, T. R. Lemberger, and J. M. Graybeal, Phys. Rev. B 63, 174505 (2001). 